\title{
JORDAN ISOMORPHISMS OF FINITARY INCIDENCE ALGEBRAS
}

\author{
ROSALI BRUSAMARELLO, ÉRICA Z. FORNAROLI, AND MYKOLA KHRYPCHENKO
}

\begin{abstract}
Let $X$ be a partially ordered set, $R$ a commutative 2-torsionfree unital ring and $F I(X, R)$ the finitary incidence algebra of $X$ over $R$. In this note we prove that each $R$-linear Jordan isomorphism of $F I(X, R)$ onto an $R$-algebra $A$ is the near-sum of a homomorphism and an anti-homomorphism.
\end{abstract}

\section{INTRODUCTION}

The study of Jordan maps between rings originated in 1940's in the works by G. Ancochea [2, 3], I. Kaplansky [12] and L. K. Hua [10]. In 1950's the topic was further developed by N. Jacobson and C. E. Rickart in [11, by I. N. Herstein in [9] and by M. F. Smiley in [18. The description of Jordan homomorphisms turned out to be closely related to the description of homomorphisms and anti-homomorphisms. In particular, Jacobson and Rickart proved in [11, Theorem 7] that, for $n \geq 2$, every Jordan homomorphism of the ring $M_{n}(R)$ of all $n \times n$-matrices over a ring $R$ into an arbitrary ring is the sum of a homomorphism and an anti-homomorphism. The classical theorem by Herstein says that every Jordan homomorphism of a ring onto a prime ring of characteristic different from 2 and 3 is either a homomorphism or an anti-homomorphism (see [9, Theorem H]). Smiley improved in [18 Herstein's result by eliminating the restriction that the characteristic be different from 3. For further generalizations see the works of W. E. Baxter and W. S. Martindale III 4, M. Brešar [7, 8, W. S. Martindale III [14, 15, K. McCrimmon [16.

L. Molnár and P. Šemrl 17] initiated the investigation of Jordan maps on the ring $T_{n}(\mathcal{C})$ of upper triangular matrices. They proved in [17, Corollary 4] that each Jordan automorphism of $T_{n}(\mathcal{C})$ is either an automorphism, or an anti-automorphism, where $\mathcal{C}$ is a field with at least 3 elements. K. I. Beidar, M. Brešar and M. A. Chebotar generalized this result in [5] by showing that each Jordan isomorphism of $T_{n}(\mathcal{C})$ onto a $\mathcal{C}$-algebra is either an isomorphism, or an anti-isomorphism, provided that $\mathcal{C}$ is a 2 -torsionfree unital commutative ring without non-trivial idempotents and $n \geq 2$. D. Benkovič introduced in [6] the notion of a near-sum, which helped him to describe in [6. Theorem 4.1] all Jordan homomorphisms $T_{n}(\mathcal{C}) \rightarrow A$, where $\mathcal{C}$ is an arbitrary 2-torsionfree commutative ring and $A$ is a $\mathcal{C}$-algebra. E. Akkurt, M. Akkurt and G. P. Barker [1] extended Benkovič's result to structural matrix algebras $T_{n}(\mathcal{C}, \rho)$, where $\rho$ is either a partial order, or a quasi-order each of whose equivalence classes contains at least 2 elements (see [1, Theorem 2.1]).

Observe that $T_{n}(\mathcal{C}, \rho)$ is isomorphic to the incidence algebra of the ordered set $(\{1, \ldots, n\}, \rho)$ over the $\operatorname{ring} \mathcal{C}$. In this paper we partially generalize the result by

2010 Mathematics Subject Classification. Primary 16S50, 17C50; Secondary 16W10.

Key words and phrases. Jordan isomorphism, near-sum, homomorphism, anti-homomorphism, finitary incidence algebra. 
E. Akkurt et al. to the case of finitary incidence algebras $F I(X, R)$, namely, we show that each $R$-linear Jordan isomorphism of $F I(X, R)$ onto an $R$-algebra $A$ is the near-sum of a homomorphism and an anti-homomorphism, where $X$ is an arbitrary partially ordered set and $R$ a commutative 2-torsionfree unital ring.

The article is organized as follows. After giving all the necessary background information on Jordan homomorphisms and (finitary) incidence algebras in Section 1, we proceed with some technical lemmas in Section 2 which allow us to prove in Proposition 2.3 that each Jordan isomorphism $\varphi: F I(X, R) \rightarrow A$ restricts to a homomorphism (and an anti-homomorphism) of the commutative subalgebra $D(X, R)$ consisting of the so-called diagonal elements. Furthermore, restricting $\varphi$ to the subalgebra $\tilde{I}(X, R)$ generated by the matrix units (7), one may directly apply the argument from the proof of [1, Theorem 2.1] to decompose $\left.\varphi\right|_{\tilde{I}(X, R)}$ into the near sum $\psi+\theta$, where $\psi$ is a homomorphism $\tilde{I}(X, R) \rightarrow A$ and $\theta$ is an antihomomorphism $\tilde{I}(X, R) \rightarrow A$. We show in Proposition 2.11 that $\psi$ extends to a homomorphism $\tilde{\psi}: F I(X, R) \rightarrow A$, and an analogous fact is proved for $\theta$ in Proposition 2.12 skipping some details. Our main result is Theorem 2.13 which says that $\tilde{\psi}+\tilde{\theta}$ is a decomposition of $\varphi$ into a near-sum.

\section{Preliminaries}

1.1. Jordan homomorphisms. Let $A$ and $B$ be algebras over a commutative ring $R$. An $R$-linear map $\varphi: A \rightarrow B$ is called a Jordan homomorphism, if it satisfies

$$
\begin{aligned}
\varphi\left(a^{2}\right) & =\varphi(a)^{2}, \\
\varphi(a b a) & =\varphi(a) \varphi(b) \varphi(a),
\end{aligned}
$$

for all $a, b \in A$. A bijective Jordan homomorphism is called a Jordan isomorphism.

Each homomorphism, as well as an anti-homomorphism, is a Jordan homomorphism. The sum of a homomorphism $\psi: A \rightarrow B$ and an anti-homomorphism $\theta: A \rightarrow B$ is a Jordan homomorphism, if $\psi(a) \theta(b)=\theta(a) \psi(b)=0$ for all $a, b \in A$. A more general construction was introduced by D. Benkovič in [6]. Suppose that $A$ can be represented as the direct sum of $R$-subspaces $A_{0} \oplus A_{1}$, where $A_{0}$ is a subalgebra of $A$ and $A_{1}$ is an ideal of $A$. Let $\psi: A \rightarrow B$ be a homomorphism and $\theta: A \rightarrow B$ an anti-homomorphism, such that $\left.\psi\right|_{A_{0}}=\left.\theta\right|_{A_{0}}$ and $\psi(a) \theta(b)=\theta(a) \psi(b)=0$ for all $a, b \in A_{1}$. Then the near-sum of $\psi$ and $\theta$ (with respect to $A_{0}$ and $A_{1}$ ) is the $R$-linear map $\varphi: A \rightarrow B$, which satisfies $\left.\varphi\right|_{A_{0}}=\left.\psi\right|_{A_{0}}=\left.\theta\right|_{A_{0}}$ and $\left.\varphi\right|_{A_{1}}=\left.\psi\right|_{A_{1}}+\left.\theta\right|_{A_{1}}$. One can show that $\varphi$ is a Jordan homomorphism in this case.

Notice that the substitution of $a$ by $a+c$ in (2) gives

$$
\varphi(a b c+c b a)=\varphi(a) \varphi(b) \varphi(c)+\varphi(c) \varphi(b) \varphi(a) .
$$

We shall also use the following fact (see Corollary 2 of [11, Theorem 1]). If $e$ is an idempotent, such that $e a=a e$, then

$$
\varphi(a) \varphi(e)=\varphi(e) \varphi(a)=\varphi(a e) .
$$

In particular, if $A$ has identity 1 , then $\varphi(1)$ is the identity of $\varphi(A)$. Another particular case of (4): if $e a=a e=0$, then

$$
\varphi(e) \varphi(a)=\varphi(a) \varphi(e)=0 .
$$


1.2. Finitary incidence algebras. Let $(X, \leq)$ be a partially ordered set and $R$ a commutative ring with identity. The incidence space $I(X, R)$ of $X$ over $R$ is defined to be the set of functions $f: X \times X \rightarrow R$, such that $f(x, y)=0$ if $x \not \leq y$, with the natural structure of an $R$-module. An element $f \in I(X, R)$, such that for any interval $[x, y] \subseteq X$ there exists only a finite number of $[u, v] \subseteq[x, y]$ with $u<v$ and $f(u, v) \neq 0$, is called a finitary series [13. The subset $F I(X, R)$ of finitary series is clearly an $R$-submodule of $I(X, R)$. Moreover, for any pair of elements $f, g \in I(X, R)$, at least one of which belongs to $F I(X, R)$, one can define the product $f g$ as the convolution

$$
(f g)(x, y)=\sum_{x \leq z \leq y} f(x, z) g(z, y)
$$

so that $F I(X, R)$ becomes an $R$-algebra, called the finitary incidence algebra of $X$ over $R$, and $I(X, R)$ a bimodule over $F I(X, R)$ (see [13, Theorem 1]). The identity element $\delta$ of $F I(X, R)$ is the function $\delta(x, y)=\delta_{x y}$ for $x \leq y$, where $\delta_{x y} \in\{0,1\}$ is the Kronecker delta. Observe that, when $X$ is locally finite, $F I(X, R)=I(X, R)$ is the (classical) incidence algebra 19 .

An element $f \in F I(X, R)$ is said to be diagonal, if $f(x, y)=0$ for $x \neq y$. Diagonal elements form a commutative subalgebra of $F I(X, R)$, which we denote by $D(X, R)$. We shall also work with $f \in I(X, R)$ satisfying $f(x, y)=0$ for $x=$ $y$. Such elements form an $F I(X, R)$-submodule of $I(X, R)$ denoted by $Z(X, R)$. Clearly, each $f \in I(X, R)$ can be uniquely written as $f=f_{D}+f_{Z}$ with $f_{D} \in$ $D(X, R)$ and $f_{Z} \in Z(X, R)$, so $I(X, R)=D(X, R) \oplus Z(X, R)$ as a module over $R$. Consequently, $F Z(X, R):=Z(X, R) \cap F I(X, R)$ is an ideal of $F I(X, R)$ and $F I(X, R)=D(X, R) \oplus F Z(X, R)$ as an $R$-module.

For each pair $x \leq y$ define $e_{x y} \in F I(X, R)$ by

$$
e_{x y}(u, v)= \begin{cases}1, & \text { if } u=x \text { and } v=y \\ 0, & \text { otherwise }\end{cases}
$$

Then $e_{x y} e_{u v}=\delta_{y u} e_{x v}$ by the definition of convolution. In particular, the elements $e_{x}:=e_{x x} \in D(X, R), x \in X$, are pairwise orthogonal idempotents of $F I(X, R)$. Observe that for any $f \in I(X, R)$

$$
e_{x} f e_{y}= \begin{cases}f(x, y) e_{x y}, & \text { if } x \leq y \\ 0, & \text { otherwise }\end{cases}
$$

Consequently,

$$
\begin{aligned}
f=g & \Leftrightarrow \forall x \leq y: \quad e_{x} f e_{y}=e_{x} g e_{y} \\
& \Leftrightarrow \begin{cases}\forall x<y: & e_{x} f e_{y}+e_{y} f e_{x}=e_{x} g e_{y}+e_{y} g e_{x}, \\
\forall x: & e_{x} f e_{x}=e_{x} g e_{x} .\end{cases}
\end{aligned}
$$

The subalgebra of $F I(X, R)$ generated by the functions $e_{x y}$ will be denoted by $\tilde{I}(X, R)$. As an $R$-module, it admits the decomposition $\tilde{I}(X, R)=\tilde{D}(X, R) \oplus$ $\tilde{Z}(X, R)$, where $\tilde{D}(X, R)=\tilde{I}(X, R) \cap D(X, R)$ is a subalgebra of $\tilde{I}(X, R)$ and $\tilde{Z}(X, R)=\tilde{I}(X, R) \cap Z(X, R)$ is an ideal of $\tilde{I}(X, R)$. 
Given $Y \subseteq X$, we introduce the notation $e_{Y}$ for the diagonal idempotent defined by

$$
e_{Y}(u, v)= \begin{cases}1, & \text { if } u=v \in Y \\ 0, & \text { otherwise. }\end{cases}
$$

In particular, $e_{x}=e_{\{x\}}$. Note that $e_{Y} e_{Z}=e_{Y \cap Z}$, so $e_{x} e_{Y}=e_{x}$ for $x \in Y$, and $e_{x} e_{Y}=0$ otherwise.

\section{JoRDAN ISOMORPHISMS OF $F I(X, R)$}

In all what follows we assume that $(X, \leq)$ is an arbitrary (non-necessarily locally finite) partially ordered set, $R$ is a commutative 2-torsionfree unital $\operatorname{ring}$ and $A$ is an associative $R$-algebra.

Let $\varphi$ be a Jordan homomorphism from $F I(X, R)$ to $A$. Then its restriction to $\tilde{I}(X, R)$ is a Jordan homomorphism $\tilde{I}(X, R) \rightarrow A$. Following the proof of Theorem 2.1 from [1, one sees that the $R$-linear maps

$$
\begin{aligned}
\psi\left(e_{x y}\right) & =\varphi\left(e_{x}\right) \varphi\left(e_{x y}\right) \varphi\left(e_{y}\right), \\
\theta\left(e_{x y}\right) & =\varphi\left(e_{y}\right) \varphi\left(e_{x y}\right) \varphi\left(e_{x}\right)
\end{aligned}
$$

are, respectively, a homomorphism and an anti-homomorphism $\tilde{I}(X, R) \rightarrow A$. Moreover, $\left.\varphi\right|_{\tilde{I}(X, R)}$ is the near-sum of $\psi$ and $\theta$ with respect to the subalgebra $\tilde{D}(X, R)$ and the ideal $\tilde{Z}(X, R)$ of $\tilde{I}(X, R)$.

When $\varphi$ is bijective, our aim is to show that $\psi$ and $\theta$ extend to a homomorphism $\tilde{\psi}$ and an anti-homomorphism $\tilde{\theta}$ from $F I(X, R)$ to $A$ in such a way that $\varphi$ is the near-sum $\tilde{\psi}+\tilde{\theta}$ with respect to $D(X, R)$ and $F Z(X, R)$.

\subsection{The restriction of $\varphi$ to $D(X, R)$.}

Lemma 2.1. Let $\varphi: F I(X, R) \rightarrow A$ be a Jordan homomorphism. Then for any $f \in F I(X, R)$ one has

$$
\begin{aligned}
\forall x<y: \quad f(x, y) \varphi\left(e_{x y}\right) & =\varphi\left(e_{x}\right) \varphi(f) \varphi\left(e_{y}\right)+\varphi\left(e_{y}\right) \varphi(f) \varphi\left(e_{x}\right), \\
\forall x: \quad f(x, x) \varphi\left(e_{x}\right) & =\varphi\left(e_{x}\right) \varphi(f) \varphi\left(e_{x}\right) .
\end{aligned}
$$

Proof. Observe from (8) that $f(x, y) e_{x y}=e_{x} f e_{y}+e_{y} f e_{x}$ for $x<y$. Therefore, (12) follows from (3). Similarly (13) is explained by (2) and (8).

Lemma 2.2. Let $\varphi: F I(X, R) \rightarrow A$ be a Jordan isomorphism. Given $a, b \in A$, one has

$$
a=b \Leftrightarrow \begin{cases}\forall x<y: & \varphi\left(e_{x}\right) a \varphi\left(e_{y}\right)+\varphi\left(e_{y}\right) a \varphi\left(e_{x}\right)=\varphi\left(e_{x}\right) b \varphi\left(e_{y}\right)+\varphi\left(e_{y}\right) b \varphi\left(e_{x}\right), \\ \forall x: & \varphi\left(e_{x}\right) a \varphi\left(e_{x}\right)=\varphi\left(e_{x}\right) b \varphi\left(e_{x}\right) .\end{cases}
$$

Proof. The result follows from (2), (3) and (9) and the bijectivity of $\varphi$.

Proposition 2.3. Let $\varphi: F I(X, R) \rightarrow A$ be a Jordan isomorphism. Then $\left.\varphi\right|_{D(X, R)}$ is a homomorphism (and an anti-homomorphism at the same time).

Proof. We shall use Lemma 2.2 to prove that $\varphi(f g)=\varphi(f) \varphi(g)$ for all $f, g \in$ $D(X, R)$. Let $x<y$ be arbitrary elements of $X$. Since the idempotents $e_{x}, e_{y}$ belong to $D(X, R)$, and $D(X, R)$ is commutative, it follows from (4) and (5) that

$$
\begin{aligned}
\varphi\left(e_{x}\right) \varphi(f g) \varphi\left(e_{y}\right) & =\varphi\left(e_{y}\right) \varphi(f g) \varphi\left(e_{x}\right)=\varphi(f g) \varphi\left(e_{x}\right) \varphi\left(e_{y}\right)=0, \\
\varphi\left(e_{x}\right) \varphi(f) \varphi(g) \varphi\left(e_{y}\right) & =\varphi\left(e_{y}\right) \varphi(f) \varphi(g) \varphi\left(e_{x}\right)=\varphi(f) \varphi\left(e_{x}\right) \varphi\left(e_{y}\right) \varphi(g)=0 .
\end{aligned}
$$


Now by (13) we have $\varphi\left(e_{x}\right) \varphi(f g) \varphi\left(e_{x}\right)=(f g)(x, x) \varphi\left(e_{x}\right)$, and taking into account (11) and (4):

$$
\begin{aligned}
\varphi\left(e_{x}\right) \varphi(f) \varphi(g) \varphi\left(e_{x}\right) & =\varphi\left(e_{x}\right) \varphi(f) \varphi\left(e_{x}\right) \cdot \varphi\left(e_{x}\right) \varphi(g) \varphi\left(e_{x}\right) \\
& =f(x, x) \varphi\left(e_{x}\right) g(x, x) \varphi\left(e_{x}\right)=f(x, x) g(x, x) \varphi\left(e_{x}\right) .
\end{aligned}
$$

As $(f g)(x, x)=f(x, x) g(x, x)$, the result follows.

\subsection{An extension of $\psi$ to $F I(X, R)$.}

Lemma 2.4. Let $\varphi: F I(X, R) \rightarrow A$ be a Jordan homomorphism and $\psi$ be given by (10). Then for all $f \in F I(X, R)$ and $x \leq y$ :

$$
\varphi\left(e_{x}\right) \varphi(f) \varphi\left(e_{y}\right)=f(x, y) \psi\left(e_{x y}\right) .
$$

Proof. If $x=y$, then $\psi\left(e_{x}\right)=\varphi\left(e_{x}\right)$, so (14) is (13). If $x<y$, then multiply (12) by $\varphi\left(e_{y}\right)$ on the right and by $\varphi\left(e_{x}\right)$ on the left to get

$$
f(x, y) \varphi\left(e_{x}\right) \varphi\left(e_{x y}\right) \varphi\left(e_{y}\right)=\varphi\left(e_{x}\right) \varphi(f) \varphi\left(e_{y}\right) .
$$

Now, (14) follows from (10).

Proposition 2.5. Let $\varphi: F I(X, R) \rightarrow A$ be a Jordan isomorphism and $\psi$ be given by (10). Then there exists an R-linear extension $\tilde{\psi}$ of $\psi$ to the whole $F I(X, R)$. Moreover, for any $f \in F Z(X, R)$ one has

$$
\begin{aligned}
\forall x<y: \varphi\left(e_{x}\right) \tilde{\psi}(f) \varphi\left(e_{y}\right) & =\varphi\left(e_{x}\right) \varphi(f) \varphi\left(e_{y}\right), \\
\forall x<y: \varphi\left(e_{y}\right) \tilde{\psi}(f) \varphi\left(e_{x}\right) & =0 \\
\forall x: \varphi\left(e_{x}\right) \tilde{\psi}(f) \varphi\left(e_{x}\right) & =0 .
\end{aligned}
$$

Proof. Given $f \in F Z(X, R)$ and $x \leq y$, set

$$
a_{x y}=\varphi\left(e_{x}\right) \varphi(f) \varphi\left(e_{y}\right) \in A
$$

and consider $g \in I(X, R)$ defined by

$$
g(x, y)=\varphi^{-1}\left(a_{x y}\right)(x, y) .
$$

Observe that $g \in Z(X, R)$, as $a_{x x}=0$ by (13). We claim that $g \in F Z(X, R)$. Indeed, suppose that $g(u, v) \neq 0$ for an infinite number of $[u, v] \subseteq[x, y]$ with $u<v$. In view of (19) one has $\varphi^{-1}\left(a_{u v}\right) \neq 0$ and thus $a_{u v} \neq 0$. Then $f(u, v) \neq 0$ thanks to (14) and (18), which contradicts the fact that $f$ is a finitary series. We now define

$$
\tilde{\psi}(f)=\varphi(g) .
$$

In the general situation, when $f \in F I(X, R)$, write $f=f_{D}+f_{Z}$ and thus set $\tilde{\psi}(f)=\varphi\left(f_{D}\right)+\tilde{\psi}\left(f_{Z}\right)$.

To prove that $\tilde{\psi}$ is linear, consider $f, g \in F I(X, R)$ and $\alpha \in R$. Then

$$
\tilde{\psi}(\alpha f+g)=\varphi\left((\alpha f+g)_{D}\right)+\tilde{\psi}\left((\alpha f+g)_{Z}\right)=\varphi\left(\alpha f_{D}+g_{D}\right)+\tilde{\psi}\left(\alpha f_{Z}+g_{Z}\right) .
$$

Since $\varphi$ is linear, it suffices to show that $\tilde{\psi}\left(\alpha f_{1}+f_{2}\right)=\alpha \tilde{\psi}\left(f_{1}\right)+\tilde{\psi}\left(f_{2}\right)$ for $f_{1}, f_{2} \in$ $F Z(X, R)$. As above, we set

$$
\left\{\begin{array} { l } 
{ a _ { x y } ^ { \prime } = \varphi ( e _ { x } ) \varphi ( f _ { 1 } ) \varphi ( e _ { y } ) } \\
{ a _ { x y } ^ { \prime \prime } = \varphi ( e _ { x } ) \varphi ( f _ { 2 } ) \varphi ( e _ { y } ) }
\end{array} \quad \text { and } \quad \left\{\begin{array}{l}
g_{1}(x, y)=\varphi^{-1}\left(a_{x y}^{\prime}\right)(x, y) \\
g_{2}(x, y)=\varphi^{-1}\left(a_{x y}^{\prime \prime}\right)(x, y)
\end{array}\right.\right.
$$


so that $\tilde{\psi}\left(f_{1}\right)=\varphi\left(g_{1}\right)$ and $\tilde{\psi}\left(f_{2}\right)=\varphi\left(g_{2}\right)$. Now,

$$
\alpha \tilde{\psi}\left(f_{1}\right)+\tilde{\psi}\left(f_{2}\right)=\alpha \varphi\left(g_{1}\right)+\varphi\left(g_{2}\right)=\varphi\left(\alpha g_{1}+g_{2}\right)=\tilde{\psi}\left(\alpha f_{1}+f_{2}\right),
$$

where the last equality follows from

$\left(\alpha g_{1}+g_{2}\right)(x, y)=\varphi^{-1}\left(\alpha a_{x y}^{\prime}+a_{x y}^{\prime \prime}\right)(x, y)$ and $\alpha a_{x y}^{\prime}+a_{x y}^{\prime \prime}=\varphi\left(e_{x}\right) \varphi\left(\alpha f_{1}+f_{2}\right) \varphi\left(e_{y}\right)$.

Now, we prove (15) (17). Since $\varphi\left(e_{x}\right) \tilde{\psi}(f) \varphi\left(e_{x}\right)=\varphi\left(e_{x}\right) \varphi(g) \varphi\left(e_{x}\right)$ by (20), equality (17) follows from (13) and the fact that $g \in F Z(X, R)$. To prove (15) and (16), observe from (11) and (5) that for all $x<y$

$$
\begin{aligned}
& \varphi\left(e_{x}\right) a_{x y} \varphi\left(e_{y}\right)=a_{x y}, \\
& \varphi\left(e_{y}\right) a_{x y} \varphi\left(e_{x}\right)=0 .
\end{aligned}
$$

Since $\varphi^{-1}$ is a Jordan isomorphism,

$$
\begin{aligned}
\varphi^{-1}\left(a_{x y}\right) & =\varphi^{-1}\left(\varphi\left(e_{x}\right) a_{x y} \varphi\left(e_{y}\right)+\varphi\left(e_{y}\right) a_{x y} \varphi\left(e_{x}\right)\right) \\
& =e_{x} \varphi^{-1}\left(a_{x y}\right) e_{y}+e_{y} \varphi^{-1}\left(a_{x y}\right) e_{x}=\varphi^{-1}\left(a_{x y}\right)(x, y) e_{x y} .
\end{aligned}
$$

Hence, by (20) and (23)

$$
\begin{aligned}
\varphi\left(e_{x}\right) \tilde{\psi}(f) \varphi\left(e_{y}\right)+\varphi\left(e_{y}\right) \tilde{\psi}(f) \varphi\left(e_{x}\right) & =\varphi\left(e_{x}\right) \varphi(g) \varphi\left(e_{y}\right)+\varphi\left(e_{y}\right) \varphi(g) \varphi\left(e_{x}\right) \\
& =\varphi\left(e_{x} g e_{y}+e_{y} g e_{x}\right)=\varphi\left(\varphi^{-1}\left(a_{x y}\right)(x, y) e_{x y}\right) \\
& =\varphi\left(\varphi^{-1}\left(a_{x y}\right)\right)=a_{x y} .
\end{aligned}
$$

Multiplying this by $\varphi\left(e_{x}\right)$ on the left and by $\varphi\left(e_{y}\right)$ on the right and using (18) and (21) we get (15). Similarly, (22) and the multiplication of (24) by $\varphi\left(e_{y}\right)$ on the left and by $\varphi\left(e_{x}\right)$ on the right give (16).

We now show that $\tilde{\psi}$ is an extension of $\psi$, i.e. it satisfies (10). This is clearly true, when $x=y$, since $\tilde{\psi}$ coincides with $\varphi$ on $D(X, R)$. Let $x<y$, so that $e_{x y} \in F Z(X, R)$. We shall use Lemma 2.2. By (17), for any $u$,

$$
\varphi\left(e_{u}\right) \tilde{\psi}\left(e_{x y}\right) \varphi\left(e_{u}\right)=0=\varphi\left(e_{u}\right) \varphi\left(e_{x}\right) \varphi\left(e_{x y}\right) \varphi\left(e_{y}\right) \varphi\left(e_{u}\right),
$$

since either $u \neq x$ or $u \neq y$. Furthermore, by (15) and (16) for any $u<v$,

$$
\varphi\left(e_{u}\right) \tilde{\psi}\left(e_{x y}\right) \varphi\left(e_{v}\right)+\varphi\left(e_{v}\right) \tilde{\psi}\left(e_{x y}\right) \varphi\left(e_{u}\right)=\varphi\left(e_{u}\right) \varphi\left(e_{x y}\right) \varphi\left(e_{v}\right) .
$$

If $\{u, v\} \neq\{x, y\}$, then the latter is zero, since $e_{u} e_{x y}=e_{x y} e_{u}=0$ or $e_{v} e_{x y}=$ $e_{x y} e_{v}=0$. But

$$
\varphi\left(e_{u}\right) \varphi\left(e_{x}\right) \varphi\left(e_{x y}\right) \varphi\left(e_{y}\right) \varphi\left(e_{v}\right)+\varphi\left(e_{v}\right) \varphi\left(e_{x}\right) \varphi\left(e_{x y}\right) \varphi\left(e_{y}\right) \varphi\left(e_{u}\right)
$$

is also zero in this case. Since $u<v$, it remains only one case to be checked: $u=x$ and $v=y$. In this situation,

$$
\begin{aligned}
\varphi\left(e_{u}\right) \varphi\left(e_{x y}\right) \varphi\left(e_{v}\right) & =\varphi\left(e_{x}\right) \varphi\left(e_{x y}\right) \varphi\left(e_{y}\right) \\
& =\varphi\left(e_{x}\right) \varphi\left(e_{x}\right) \varphi\left(e_{x y}\right) \varphi\left(e_{y}\right) \varphi\left(e_{y}\right)+\varphi\left(e_{y}\right) \varphi\left(e_{x}\right) \varphi\left(e_{x y}\right) \varphi\left(e_{y}\right) \varphi\left(e_{x}\right) .
\end{aligned}
$$

The proof that $\tilde{\psi}$ respects multiplication will be divided into several lemmas.

Lemma 2.6. Let $\varphi: F I(X, R) \rightarrow A$ be a Jordan isomorphism and $\tilde{\psi}: F I(X, R) \rightarrow$ $A$ as defined in Proposition 2.5. If $f \in D(X, R)$ and $g \in F Z(X, R)$, then $\tilde{\psi}(f g)=$ $\tilde{\psi}(f) \tilde{\psi}(g)$. 
Proof. To prove the equality, we shall use Lemma 2.2. Let $x \leq y$. If $x<y$, then by (14) (16)

$$
\varphi\left(e_{x}\right) \tilde{\psi}(f g) \varphi\left(e_{y}\right)+\varphi\left(e_{y}\right) \tilde{\psi}(f g) \varphi\left(e_{x}\right)=(f g)(x, y) \tilde{\psi}\left(e_{x y}\right)=f(x, x) g(x, y) \tilde{\psi}\left(e_{x y}\right) .
$$

Now, we need to compute

$$
\varphi\left(e_{x}\right) \tilde{\psi}(f) \tilde{\psi}(g) \varphi\left(e_{y}\right)+\varphi\left(e_{y}\right) \tilde{\psi}(f) \tilde{\psi}(g) \varphi\left(e_{x}\right) .
$$

Since $e_{x}, f \in D(X, R), e_{x} f=f(x, x) e_{x}$ and $\left.\tilde{\psi}\right|_{D(X, R)}=\left.\varphi\right|_{D(X, R)}$ is a homomorphism, we have by (14) and (15)

$$
\begin{aligned}
\varphi\left(e_{x}\right) \tilde{\psi}(f) \tilde{\psi}(g) \varphi\left(e_{y}\right) & =\varphi\left(e_{x}\right) \varphi(f) \tilde{\psi}(g) \varphi\left(e_{y}\right)=f(x, x) \varphi\left(e_{x}\right) \tilde{\psi}(g) \varphi\left(e_{y}\right) \\
& =f(x, x) g(x, y) \tilde{\psi}\left(e_{x y}\right) .
\end{aligned}
$$

Similarly by (16)

$$
\varphi\left(e_{y}\right) \tilde{\psi}(f) \tilde{\psi}(g) \varphi\left(e_{x}\right)=f(y, y) \varphi\left(e_{y}\right) \tilde{\psi}(g) \varphi\left(e_{x}\right)=0,
$$

so (26) coincides with the last term of (25).

If $x=y$, then $\varphi\left(e_{x}\right) \tilde{\psi}(f g) \varphi\left(e_{x}\right)=0$ by (17), because $f g \in F Z(X, R)$. By the same reason

$$
\varphi\left(e_{x}\right) \tilde{\psi}(f) \tilde{\psi}(g) \varphi\left(e_{x}\right)=f(x, x) \varphi\left(e_{x}\right) \tilde{\psi}(g) \varphi\left(e_{x}\right)=0
$$

Remark 2.7. By a similar computation one proves that if $f \in F Z(X, R)$ and $g \in D(X, R)$, then $\tilde{\psi}(f g)=\tilde{\psi}(f) \tilde{\psi}(g)$.

It remains to consider the case $f, g \in F Z(X, R)$. To treat it, we shall need two technical lemmas.

Lemma 2.8. Let $\varphi: A \rightarrow B$ be a Jordan homomorphism. Given a, b, $c, d, e \in A$, one has

$$
\begin{aligned}
\varphi(a b c d e+e d a b c+c b a d e+e d c b a)= & \varphi(a) \varphi(b) \varphi(c) \varphi(d) \varphi(e)+\varphi(e) \varphi(d) \varphi(a) \varphi(b) \varphi(c) \\
& +\varphi(c) \varphi(b) \varphi(a) \varphi(d) \varphi(e)+\varphi(e) \varphi(d) \varphi(c) \varphi(b) \varphi(a) .
\end{aligned}
$$

Proof. By (3),

$$
\begin{aligned}
& \varphi((a b c) d e+e d(a b c))=\varphi(a b c) \varphi(d) \varphi(e)+\varphi(e) \varphi(d) \varphi(a b c), \\
& \varphi((c b a) d e+e d(c b a))=\varphi(c b a) \varphi(d) \varphi(e)+\varphi(e) \varphi(d) \varphi(c b a) .
\end{aligned}
$$

It remains to add these equalities and use (3) once again.

Lemma 2.9. Let $\varphi: F I(X, R) \rightarrow A$ be a Jordan isomorphism and $\tilde{\psi}: F I(X, R) \rightarrow$ $A$ as defined in Proposition 2.5. Given $f, g \in F Z(X, R)$ and $f^{\prime}, g^{\prime} \in F Z(X, R)$ such that $\tilde{\psi}(f)=\varphi\left(f^{\prime}\right)$ and $\tilde{\psi}(g)=\varphi\left(g^{\prime}\right)$, one has for all $x \leq y$ and $W \subseteq X \backslash\{z \in$ $\left.[x, y] \mid f^{\prime}(x, z) \neq 0 \neq g^{\prime}(z, y)\right\}$

$$
\varphi\left(e_{x}\right) \tilde{\psi}(f) \varphi\left(e_{W}\right) \tilde{\psi}(g) \varphi\left(e_{y}\right)=\varphi\left(e_{y}\right) \tilde{\psi}(f) \varphi\left(e_{W}\right) \tilde{\psi}(g) \varphi\left(e_{x}\right)=0 .
$$


Proof. Assume first that $x<y$. Let us apply Lemma 2.8 with $a=e_{x}, b=f^{\prime}$, $c=e_{W}, d=g^{\prime}$ and $e=e_{y}$. The products $e_{y} g^{\prime} e_{x} f^{\prime} e_{W}$ and $e_{y} g^{\prime} e_{W} f^{\prime} e_{x}$ are zero thanks to (8). The product $e_{x} f^{\prime} e_{W} g^{\prime} e_{y}$ equals $\left(f^{\prime} e_{W} g^{\prime}\right)(x, y) e_{x y}$, which is also zero by (6). Thus,

$$
\begin{aligned}
\varphi\left(e_{W} f^{\prime} e_{x} g^{\prime} e_{y}\right)= & \varphi\left(e_{x}\right) \varphi\left(f^{\prime}\right) \varphi\left(e_{W}\right) \varphi\left(g^{\prime}\right) \varphi\left(e_{y}\right) \\
& +\varphi\left(e_{y}\right) \varphi\left(g^{\prime}\right) \varphi\left(e_{x}\right) \varphi\left(f^{\prime}\right) \varphi\left(e_{W}\right) \\
& +\varphi\left(e_{W}\right) \varphi\left(f^{\prime}\right) \varphi\left(e_{x}\right) \varphi\left(g^{\prime}\right) \varphi\left(e_{y}\right) \\
& +\varphi\left(e_{y}\right) \varphi\left(g^{\prime}\right) \varphi\left(e_{W}\right) \varphi\left(f^{\prime}\right) \varphi\left(e_{x}\right) .
\end{aligned}
$$

Suppose first that $x \in W$. Multiplying (27) by $\varphi\left(e_{x}\right)$ on the left and using (5), we get zero on the left-hand side of (27), as $e_{x} e_{W} f^{\prime} e_{x}=f^{\prime}(x, x) e_{x}=0$ and $e_{y} e_{x}=0$. The second and fourth terms of the right-hand side of (27) are also zero by (5), the third one is zero by (14) and Proposition 2.3, while the first one does not change by (11). Similarly the multiplication of (27) by $\varphi\left(e_{x}\right)$ on the right gives $\varphi\left(e_{y}\right) \varphi\left(g^{\prime}\right) \varphi\left(e_{W}\right) \varphi\left(f^{\prime}\right) \varphi\left(e_{x}\right)=0$. It remains to replace $\varphi\left(f^{\prime}\right)$ by $\tilde{\psi}(f)$ and $\varphi\left(g^{\prime}\right)$ by $\tilde{\psi}(g)$. The same argument works, when $x \notin W$, since $e_{x} e_{W}=e_{W} e_{x}=0$ in this case.

Now let $x=y$. Observe that $W$ is an arbitrary subset of $X$ in this case. We first prove the following:

$$
\varphi\left(e_{x}\right) \tilde{\psi}(f) \varphi\left(e_{W}\right)=\varphi\left(e_{x}\right) \tilde{\psi}\left(f_{>x}\right) \varphi\left(e_{W}\right),
$$

where

$$
f_{>x}(u, v)= \begin{cases}f(u, v), & \text { if } u=x \text { and } v>x, \\ 0, & \text { otherwise. }\end{cases}
$$

To this end we use Lemma 2.2. Thanks to (5) and (17), for any $u$ both sides of (28) become zero after multiplication by $\varphi\left(e_{u}\right)$ on the left and on the right. Now take $u<v$ and consider

$$
\varphi\left(e_{u}\right) \varphi\left(e_{x}\right) \tilde{\psi}(f) \varphi\left(e_{W}\right) \varphi\left(e_{v}\right)+\varphi\left(e_{v}\right) \varphi\left(e_{x}\right) \tilde{\psi}(f) \varphi\left(e_{W}\right) \varphi\left(e_{u}\right)
$$

If $x \notin\{u, v\}$ or $\{u, v\} \cap W=\emptyset$, then (30) is zero for any $f$ by (5). Let $x=u<v \in$ $W$. Then the sum (30) is $\varphi\left(e_{x}\right) \tilde{\psi}(f) \varphi\left(e_{v}\right)$ by (11) and (5), which by (14) and (15) equals

$$
f(x, v) \tilde{\psi}\left(e_{x v}\right)=f_{>x}(x, v) \tilde{\psi}\left(e_{x v}\right)=\varphi\left(e_{x}\right) \tilde{\psi}\left(f_{>x}\right) \varphi\left(e_{v}\right),
$$

so $f$ can be replaced by $f_{>x}$ in (30). If $W \ni u<v=x$, then (30) becomes $\varphi\left(e_{x}\right) \tilde{\psi}(f) \varphi\left(e_{u}\right)$, which is zero for any $f \in F Z(X, R)$ by (16). If $W \ni x=u<v \notin$ $W$ or $W \not \supset u<v=x \in W$, then (30) is again zero for any $f$.

Similarly we get

$$
\varphi\left(e_{W}\right) \tilde{\psi}(f) \varphi\left(e_{x}\right)=\varphi\left(e_{W}\right) \tilde{\psi}\left(f_{<x}\right) \varphi\left(e_{x}\right),
$$

where

$$
f_{<x}(u, v)= \begin{cases}f(u, v), & \text { if } u<x \text { and } v=x, \\ 0, & \text { otherwise. }\end{cases}
$$

Therefore, using (11) we deduce from (28) and (31)

$$
\varphi\left(e_{x}\right) \tilde{\psi}(f) \varphi\left(e_{W}\right) \tilde{\psi}(g) \varphi\left(e_{x}\right)=\varphi\left(e_{x}\right) \tilde{\psi}\left(f_{>x}\right) \varphi\left(e_{W}\right) \tilde{\psi}\left(g_{<x}\right) \varphi\left(e_{x}\right) .
$$


Now write $\tilde{\psi}\left(f_{>x}\right)=\varphi\left(f^{\prime \prime}\right), \tilde{\psi}\left(g_{<x}\right)=\varphi\left(g^{\prime \prime}\right)$ for some $f^{\prime \prime}, g^{\prime \prime} \in F Z(X, R)$ and apply Lemma 2.8 with $a=e_{x}, b=f^{\prime \prime}, c=e_{W}, d=g^{\prime \prime}$ and $e=e_{x}$. The products $e_{x} g^{\prime \prime} e_{x} f^{\prime \prime} e_{W}$ and $e_{W} f^{\prime \prime} e_{x} g^{\prime \prime} e_{x}$ are zero by (8) and the fact that $g^{\prime \prime} \in F Z(X, R)$. Similarly $e_{x} f^{\prime \prime} e_{W} g^{\prime \prime} e_{x}=e_{x} g^{\prime \prime} e_{W} f^{\prime \prime} e_{x}=0$, since $f^{\prime \prime} e_{W} g^{\prime \prime}, g^{\prime \prime} e_{W} f^{\prime \prime} \in F Z(X, R)$. Hence,

$$
\begin{aligned}
0= & \varphi\left(e_{x}\right) \varphi\left(f^{\prime \prime}\right) \varphi\left(e_{W}\right) \varphi\left(g^{\prime \prime}\right) \varphi\left(e_{x}\right)+\varphi\left(e_{x}\right) \varphi\left(g^{\prime \prime}\right) \varphi\left(e_{x}\right) \varphi\left(f^{\prime \prime}\right) \varphi\left(e_{W}\right) \\
& +\varphi\left(e_{W}\right) \varphi\left(f^{\prime \prime}\right) \varphi\left(e_{x}\right) \varphi\left(g^{\prime \prime}\right) \varphi\left(e_{x}\right)+\varphi\left(e_{x}\right) \varphi\left(g^{\prime \prime}\right) \varphi\left(e_{W}\right) \varphi\left(f^{\prime \prime}\right) \varphi\left(e_{x}\right) .
\end{aligned}
$$

By (14) the second and third summands of (34) are zero, because $g^{\prime \prime} \in F Z(X, R)$. Thus,

$$
\varphi\left(e_{x}\right) \tilde{\psi}\left(f_{>x}\right) \varphi\left(e_{W}\right) \tilde{\psi}\left(g_{<x}\right) \varphi\left(e_{x}\right)+\varphi\left(e_{x}\right) \tilde{\psi}\left(g_{<x}\right) \varphi\left(e_{W}\right) \tilde{\psi}\left(f_{>x}\right) \varphi\left(e_{x}\right)=0 .
$$

But

$$
\varphi\left(e_{x}\right) \tilde{\psi}\left(g_{<x}\right) \varphi\left(e_{W}\right) \tilde{\psi}\left(f_{>x}\right) \varphi\left(e_{x}\right)=\varphi\left(e_{x}\right) \tilde{\psi}\left(\left(g_{<x}\right)_{>x}\right) \varphi\left(e_{W}\right) \tilde{\psi}\left(\left(f_{>x}\right)_{<x}\right) \varphi\left(e_{x}\right)
$$

thanks to (33), which is zero, as $\left(g_{<x}\right)_{>x}=\left(f_{>x}\right)_{<x}=0$ by (29) and (32). So,

$$
\varphi\left(e_{x}\right) \tilde{\psi}\left(f_{>x}\right) \varphi\left(e_{W}\right) \tilde{\psi}\left(g_{<x}\right) \varphi\left(e_{x}\right)=0,
$$

which in view of (33) gives the desired equality.

Lemma 2.10. Let $\varphi: F I(X, R) \rightarrow R$ be a Jordan isomorphism and $\tilde{\psi}: F I(X, R) \rightarrow$ $A$ as defined in Proposition 2.5. If $f, g \in F Z(X, R)$, then $\tilde{\psi}(f g)=\tilde{\psi}(f) \tilde{\psi}(g)$.

Proof. As in the proof of Lemma 2.6 for $x<y$ we have

$$
\varphi\left(e_{x}\right) \tilde{\psi}(f g) \varphi\left(e_{y}\right)+\varphi\left(e_{y}\right) \tilde{\psi}(f g) \varphi\left(e_{x}\right)=(f g)(x, y) \tilde{\psi}\left(e_{x y}\right),
$$

the latter being

$$
\begin{aligned}
\sum_{z \in Z} f(x, z) \tilde{\psi}\left(e_{x z}\right) g(z, y) \tilde{\psi}\left(e_{z y}\right) & =\sum_{z \in Z} \varphi\left(e_{x}\right) \varphi(f) \varphi\left(e_{z}\right) \varphi(g) \varphi\left(e_{y}\right) \\
& =\varphi\left(e_{x}\right) \varphi(f) \varphi\left(e_{Z}\right) \varphi(g) \varphi\left(e_{y}\right)
\end{aligned}
$$

where $Z$ is any finite subset of $[x, y]$ which contains $\{z \in[x, y] \mid f(x, z) \neq 0 \neq$ $g(z, y)\}$. In particular, we may take

$$
Z=\{z \in[x, y] \mid f(x, z) \neq 0 \neq g(z, y)\} \cup\left\{z \in[x, y] \mid f^{\prime}(x, z) \neq 0 \neq g^{\prime}(z, y)\right\}
$$

with $f^{\prime}, g^{\prime} \in F Z(X, R)$ being such that $\tilde{\psi}(f)=\varphi\left(f^{\prime}\right)$ and $\tilde{\psi}(g)=\varphi\left(g^{\prime}\right)$.

On the other hand,

$$
\begin{aligned}
\varphi\left(e_{x}\right) \tilde{\psi}(f) \tilde{\psi}(g) \varphi\left(e_{y}\right)= & \varphi\left(e_{x}\right) \tilde{\psi}(f) \varphi\left(e_{Z}\right) \tilde{\psi}(g) \varphi\left(e_{y}\right) \\
& +\varphi\left(e_{x}\right) \tilde{\psi}(f) \varphi\left(e_{X \backslash Z}\right) \tilde{\psi}(g) \varphi\left(e_{y}\right), \\
\varphi\left(e_{y}\right) \tilde{\psi}(f) \tilde{\psi}(g) \varphi\left(e_{x}\right)= & \varphi\left(e_{y}\right) \tilde{\psi}(f) \varphi\left(e_{Z}\right) \tilde{\psi}(g) \varphi\left(e_{x}\right) \\
& +\varphi\left(e_{y}\right) \tilde{\psi}(f) \varphi\left(e_{X \backslash Z}\right) \tilde{\psi}(g) \varphi\left(e_{x}\right) .
\end{aligned}
$$

By Lemma 2.9 the second summands of the right-hand sides of (36) and (37) are zero. Moreover,

$$
\varphi\left(e_{y}\right) \tilde{\psi}(f) \varphi\left(e_{Z}\right) \tilde{\psi}(g) \varphi\left(e_{x}\right)=\sum_{z \in Z} \varphi\left(e_{y}\right) \tilde{\psi}(f) \varphi\left(e_{z}\right) \tilde{\psi}(g) \varphi\left(e_{x}\right)=0,
$$


because $\varphi\left(e_{z}\right) \tilde{\psi}(g) \varphi\left(e_{x}\right)=0$ for all $x \leq z$ by (16) and (17). Finally, by (15)

$$
\begin{aligned}
\varphi\left(e_{x}\right) \tilde{\psi}(f) \varphi\left(e_{Z}\right) \tilde{\psi}(g) \varphi\left(e_{y}\right) & =\sum_{z \in Z} \varphi\left(e_{x}\right) \varphi(f) \varphi\left(e_{z}\right) \varphi(g) \varphi\left(e_{y}\right) \\
& =\varphi\left(e_{x}\right) \varphi(f) \varphi\left(e_{Z}\right) \varphi(g) \varphi\left(e_{y}\right),
\end{aligned}
$$

proving that

$\varphi\left(e_{x}\right) \tilde{\psi}(f g) \varphi\left(e_{y}\right)+\varphi\left(e_{y}\right) \tilde{\psi}(f g) \varphi\left(e_{x}\right)=\varphi\left(e_{x}\right) \tilde{\psi}(f) \tilde{\psi}(g) \varphi\left(e_{y}\right)+\varphi\left(e_{y}\right) \tilde{\psi}(f) \tilde{\psi}(g) \varphi\left(e_{x}\right)$.

Now using the fact that $f g \in F Z(X, R)$ and (17), we get

$$
\varphi\left(e_{x}\right) \tilde{\psi}(f g) \varphi\left(e_{x}\right)=0 \text {. }
$$

On the other hand,

$$
\varphi\left(e_{x}\right) \tilde{\psi}(f) \tilde{\psi}(g) \varphi\left(e_{x}\right)=\varphi\left(e_{x}\right) \tilde{\psi}(f) \varphi(\delta) \tilde{\psi}(g) \varphi\left(e_{x}\right)=\varphi\left(e_{x}\right) \tilde{\psi}(f) \varphi\left(e_{X}\right) \tilde{\psi}(g) \varphi\left(e_{x}\right),
$$

which is zero thanks to Lemma 2.9. The result now follows from Lemma 2.2 .

Proposition 2.11. Let $\varphi: F I(X, R) \rightarrow R$ be a Jordan isomorphism and $\tilde{\psi}:$ $F I(X, R) \rightarrow A$ as defined in Proposition 2.5. Then $\tilde{\psi}$ is a homomorphism.

Proof. Consider $f, g \in F I(X, R)$ and write $f=f_{D}+f_{Z}$ and $g=g_{D}+g_{Z}$. Using the definition of $\tilde{\psi}$, its linearity, Lemmas 2.6 and 2.10, Remark 2.7, and Proposition 2.3. we get

$$
\begin{aligned}
\tilde{\psi}(f g) & =\tilde{\psi}\left(\left(f_{D}+f_{Z}\right)\left(g_{D}+g_{Z}\right)\right) \\
& =\tilde{\psi}\left(f_{D} g_{D}+\left(f_{D} g_{Z}+f_{Z} g_{D}+f_{Z} g_{Z}\right)\right) \\
& =\varphi\left(f_{D} g_{D}\right)+\tilde{\psi}\left(f_{D} g_{Z}+f_{Z} g_{D}+f_{Z} g_{Z}\right) \\
& =\varphi\left(f_{D}\right) \varphi\left(g_{D}\right)+\tilde{\psi}\left(f_{D} g_{Z}\right)+\tilde{\psi}\left(f_{Z} g_{D}\right)+\tilde{\psi}\left(f_{Z} g_{Z}\right) \\
& =\varphi\left(f_{D}\right) \varphi\left(g_{D}\right)+\tilde{\psi}\left(f_{D}\right) \tilde{\psi}\left(g_{Z}\right)+\tilde{\psi}\left(f_{Z}\right) \tilde{\psi}\left(g_{D}\right)+\tilde{\psi}\left(f_{Z}\right) \tilde{\psi}\left(g_{Z}\right) \\
& =\varphi\left(f_{D}\right) \varphi\left(g_{D}\right)+\varphi\left(f_{D}\right) \tilde{\psi}\left(g_{Z}\right)+\tilde{\psi}\left(f_{Z}\right) \varphi\left(g_{D}\right)+\tilde{\psi}\left(f_{Z}\right) \tilde{\psi}\left(g_{Z}\right) \\
& =\left(\varphi\left(f_{D}\right)+\tilde{\psi}\left(f_{Z}\right)\right)\left(\varphi\left(g_{D}\right)+\tilde{\psi}\left(g_{Z}\right)\right) \\
& =\tilde{\psi}(f) \tilde{\psi}(g) .
\end{aligned}
$$

2.3. A decomposition of $\varphi$ into a near-sum. Similarly to what was done in Proposition 2.5 for $\psi$, one can extend $\theta$ defined in (11) to an anti-homomorphism $F I(X, R) \rightarrow A$.

Proposition 2.12. Let $\varphi: F I(X, R) \rightarrow A$ be a Jordan isomorphism and $\theta$ be given by (11). Then there exists an extension $\tilde{\theta}$ of $\theta$ to an anti-homomorphism $F I(X, R) \rightarrow A$. Moreover, for any $f \in F Z(X, R)$ one has

$$
\begin{aligned}
\forall x<y: \varphi\left(e_{y}\right) \tilde{\theta}(f) \varphi\left(e_{x}\right) & =\varphi\left(e_{y}\right) \varphi(f) \varphi\left(e_{x}\right), \\
\forall x<y: \varphi\left(e_{x}\right) \tilde{\theta}(f) \varphi\left(e_{y}\right) & =0 \\
\forall x: \varphi\left(e_{x}\right) \tilde{\theta}(f) \varphi\left(e_{x}\right) & =0 .
\end{aligned}
$$

Proof. Given $f \in F Z(X, R)$ and $x \leq y$, set

$$
b_{x y}=\varphi\left(e_{y}\right) \varphi(f) \varphi\left(e_{x}\right) \in A
$$


and define $h \in I(X, R)$ by

$$
h(x, y)=\varphi^{-1}\left(b_{x y}\right)(x, y),
$$

for all $x \leq y$. Observe as in the proof of Proposition 2.5 that $h \in F Z(X, R)$. Define

$$
\tilde{\theta}(f)=\varphi(h) .
$$

In the general situation, when $f \in F I(X, R)$, write $f=f_{D}+f_{Z}$ and thus set $\tilde{\theta}(f)=\varphi\left(f_{D}\right)+\tilde{\theta}\left(f_{Z}\right)$.

The proof that $\tilde{\theta}$ is an anti-homomorphism satisfying (38) (40) is analogous to what was done for $\tilde{\psi}$ (see Propositions 2.5 and 2.11, Lemmas 2.6, 2.9 and 2.10, and Remark 2.7).

Finally, we are ready to prove the main result of this paper.

Theorem 2.13. Each Jordan isomorphism $\varphi: F I(X, R) \rightarrow A$ is the near-sum of $\tilde{\psi}$ and $\tilde{\theta}$ with respect to the subalgebra $D(X, R)$ and the ideal $F Z(X, R)$, where $\tilde{\psi}$ and $\tilde{\theta}$ are defined in Propositions [2.5 and 2.12, respectively.

Proof. By definition, $\left.\tilde{\psi}\right|_{D(X, R)}=\left.\tilde{\theta}\right|_{D(X, R)}=\left.\varphi\right|_{D(X, R)}$. Now, for $f \in F Z(X, R)$, $\tilde{\psi}(f)+\tilde{\theta}(f)=\varphi(g)+\varphi(h)=\varphi(g+h)$, where $g$ and $h$ are defined in (19) and (41). Since $\varphi^{-1}$, being a Jordan isomorphism, satisfies (3), for all $x \leq y$,

$$
\begin{aligned}
(g+h)(x, y) & =g(x, y)+h(x, y)=\varphi^{-1}\left(a_{x y}\right)(x, y)+\varphi^{-1}\left(b_{x y}\right)(x, y) \\
& =\left[\varphi^{-1}\left(a_{x y}\right)+\varphi^{-1}\left(b_{x y}\right)\right](x, y)=\varphi^{-1}\left(a_{x y}+b_{x y}\right)(x, y) \\
& =\left[\varphi^{-1}\left(\varphi\left(e_{x}\right) \varphi(f) \varphi\left(e_{y}\right)+\varphi\left(e_{y}\right) \varphi(f) \varphi\left(e_{x}\right)\right)\right](x, y) \\
& =\left[e_{x} f e_{y}+e_{y} f e_{x}\right](x, y)=f(x, y) .
\end{aligned}
$$

Thus $\varphi(f)=\tilde{\psi}(f)+\tilde{\theta}(f)$, for all $f \in F Z(X, R)$, i. e. $\left.\varphi\right|_{F Z(X, R)}=\left.\tilde{\psi}\right|_{F Z(X, R)}+$ $\left.\tilde{\theta}\right|_{F Z(X, R)}$.

It remains to check that the products $\tilde{\psi}(f) \tilde{\theta}\left(f^{\prime}\right)$ and $\tilde{\theta}\left(f^{\prime}\right) \tilde{\psi}(f)$ are zero for all $f, f^{\prime} \in F Z(X, R)$. By symmetry, it is enough to show that $\tilde{\psi}(f) \tilde{\theta}\left(f^{\prime}\right)=0$. To this end we use Lemma 2.2 .

Let $x<y$. Using the fact that $\tilde{\psi}(f)=\varphi(g)$ and $\tilde{\theta}\left(f^{\prime}\right)=\varphi\left(h^{\prime}\right)$, where $g$ and $h^{\prime}$ are defined in (19) and (41), we represent

$$
\varphi\left(e_{x}\right) \tilde{\psi}(f) \tilde{\theta}\left(f^{\prime}\right) \varphi\left(e_{y}\right)+\varphi\left(e_{y}\right) \tilde{\psi}(f) \tilde{\theta}\left(f^{\prime}\right) \varphi\left(e_{x}\right)
$$

as

$$
\begin{aligned}
& \sum_{z \in Z} \varphi\left(e_{x}\right) \varphi(g) \varphi\left(e_{z}\right) \varphi\left(h^{\prime}\right) \varphi\left(e_{y}\right)+\varphi\left(e_{x}\right) \varphi(g) \varphi\left(e_{X \backslash Z}\right) \varphi\left(h^{\prime}\right) \varphi\left(e_{y}\right) \\
& \quad+\sum_{z \in Z} \varphi\left(e_{y}\right) \varphi(g) \varphi\left(e_{z}\right) \varphi\left(h^{\prime}\right) \varphi\left(e_{x}\right)+\varphi\left(e_{y}\right) \varphi(g) \varphi\left(e_{X \backslash Z}\right) \varphi\left(h^{\prime}\right) \varphi\left(e_{x}\right),
\end{aligned}
$$

where $Z$ is a finite subset of $[x, y]$ which contains $\{z \in[x, y] \mid f(x, z) \neq 0 \neq g(z, y)\}$. As in the proof of Lemma 2.9 the second and fourth summands of (43) are zero. Moreover, $\varphi\left(e_{z}\right) \varphi\left(h^{\prime}\right) \varphi\left(e_{y}\right)=\varphi\left(e_{z}\right) \tilde{\theta}\left(f^{\prime}\right) \varphi\left(e_{y}\right)=0$ for all $z \in Z$ thanks to (39) and (40). Similarly, $\varphi\left(e_{y}\right) \varphi(g) \varphi\left(e_{z}\right)=\varphi\left(e_{y}\right) \tilde{\psi}(f) \varphi\left(e_{z}\right)=0$ for all $z \in Z$ in view of (16) and (17). Therefore, the first and third summands of (43) are also zero, yielding that (42) equals 0 . 
Now take $x \in X$ and write

$$
\varphi\left(e_{x}\right) \tilde{\psi}(f) \tilde{\theta}\left(f^{\prime}\right) \varphi\left(e_{x}\right)=\varphi\left(e_{x}\right) \tilde{\psi}(f) \varphi\left(e_{X}\right) \tilde{\theta}\left(f^{\prime}\right) \varphi\left(e_{x}\right) .
$$

To prove that the right-hand side of (44) is zero, we observe as in the proof of Lemma 2.9 that

$$
\begin{aligned}
& \varphi\left(e_{x}\right) \tilde{\theta}\left(f^{\prime}\right) \varphi\left(e_{X}\right)=\varphi\left(e_{x}\right) \tilde{\theta}\left(f_{<x}^{\prime}\right) \varphi\left(e_{X}\right), \\
& \varphi\left(e_{X}\right) \tilde{\theta}\left(f^{\prime}\right) \varphi\left(e_{x}\right)=\varphi\left(e_{X}\right) \tilde{\theta}\left(f_{>x}^{\prime}\right) \varphi\left(e_{x}\right)
\end{aligned}
$$

(see (28) and (31)). It follows from (28) and (46) that

$$
\varphi\left(e_{x}\right) \tilde{\psi}(f) \varphi\left(e_{X}\right) \tilde{\theta}\left(f^{\prime}\right) \varphi\left(e_{x}\right)=\varphi\left(e_{x}\right) \tilde{\psi}\left(f_{>x}\right) \varphi\left(e_{X}\right) \tilde{\theta}\left(f_{>x}^{\prime}\right) \varphi\left(e_{x}\right) .
$$

Similarly (31) and (45) yield

$$
\varphi\left(e_{x}\right) \tilde{\theta}\left(f^{\prime}\right) \varphi\left(e_{X}\right) \tilde{\psi}(f) \varphi\left(e_{x}\right)=\varphi\left(e_{x}\right) \tilde{\theta}\left(f_{<x}^{\prime}\right) \varphi\left(e_{X}\right) \tilde{\psi}\left(f_{<x}\right) \varphi\left(e_{x}\right) .
$$

Observe as in (35) that

$$
\varphi\left(e_{x}\right) \tilde{\psi}\left(f_{>x}\right) \varphi\left(e_{X}\right) \tilde{\theta}\left(f_{>x}^{\prime}\right) \varphi\left(e_{x}\right)+\varphi\left(e_{x}\right) \tilde{\theta}\left(f_{>x}^{\prime}\right) \varphi\left(e_{X}\right) \tilde{\psi}\left(f_{>x}\right) \varphi\left(e_{x}\right)=0 .
$$

But $\varphi\left(e_{x}\right) \tilde{\theta}\left(f_{>x}^{\prime}\right) \varphi\left(e_{X}\right) \tilde{\psi}\left(f_{>x}\right) \varphi\left(e_{x}\right)$ equals

$$
\varphi\left(e_{x}\right) \tilde{\theta}\left(\left(f_{>x}^{\prime}\right)_{<x}\right) \varphi\left(e_{X}\right) \tilde{\psi}\left(\left(f_{>x}\right)_{<x}\right) \varphi\left(e_{x}\right)=0
$$

in view of (29), (32) and (48). Thus, $\varphi\left(e_{x}\right) \tilde{\psi}(f) \varphi\left(e_{X}\right) \tilde{\theta}\left(f^{\prime}\right) \varphi\left(e_{x}\right)=0$ by (47) and (49), as desired.

\section{ACKNOWLEDGMENTS}

The first and second authors were partially supported by Fundação Araucária, Convênio 212/14. The third author thanks the Department of Mathematics of Maringá State University for its warm hospitality and financial support.

\section{REFERENCES}

[1] Akkurt, E., Akkurt, M., And Barker, G. P. Jordan homomorphisms of the structural matrix algebras. Linear Multilinear Algebra 63, 12 (2015), 2518-2525.

[2] Ancochea, G. Le théorème de von Staudt en géométrie projective quaternionienne. J. Reine Angew. Math. 184 (1942), 193-198.

[3] Ancochea, G. On semi-automorphisms of division algebras. Ann. Math. (2) 48 (1947), 147153.

[4] Baxter, W. E., and Martindale III, W. S. Jordan homomorphisms of semiprime rings. J. Algebra 56 (1979), 457-471.

[5] Beidar, K. I., Brešar, M., And Chebotar, M. A. Jordan isomorphisms of triangular matrix algebras over a connected commutative ring. Linear Algebra Appl. 312, 1-3 (2000), 197-201.

[6] Benkovič, D. Jordan homomorphisms on triangular matrices. Linear Multilinear Algebra 53, 5 (2005), 345-356.

[7] BREšAR, M. Jordan mappings of semiprime rings. J. Algebra 127, 1 (1989), 218-228.

[8] BrešAr, M. Jordan mappings of semiprime rings II. Bull. Aust. Math. Soc. 44, 2 (1991), 233-238.

[9] Herstein, I. N. Jordan homomorphisms. Trans. Am. Math. Soc. 81 (1956), 331-341.

[10] Hua, L. K. On the automorphisms of a field. Proc. Nat. Acad. Sci. U.S.A. 35 (1949), 386389.

[11] Jacobson, N., And Rickart, C. E. Jordan homomorphisms of rings. Trans. Amer. Math. Soc. 69 (1950), 479-502.

[12] Kaplansky, I. Semi-automorphisms of rings. Duke Math. J. 14 (1947), 521-525.

[13] Khripchenko, N. S., And Novikov, B. V. Finitary incidence algebras. Comm. Algebra 37, 5 (2009), 1670-1676. 
[14] Martindale III, W. S. Jordan homomorphisms of the symmetric elements of a ring with involution. J. Algebra 5 (1967), 232-249.

[15] Martindale III, W. S. Jordan homomorphisms onto nondegenerate Jordan algebras. J. Algebra 133, 2 (1990), 500-511.

[16] McCrimmon, K. The Zelmanov approach to Jordan homomorphisms of associative algebras. J. Algebra 123, 2 (1989), 457-477.

[17] Molnár, L., ANd ŠEmrl, P. Some linear preserver problems on upper triangular matrices. Linear Multilinear Algebra 45, 2-3 (1998), 189-206.

[18] Smiley, M. F. Jordan homomorphisms onto prime rings. Trans. Am. Math. Soc. 84 (1957), $426-429$

[19] Spiegel, E., And O'Donnell, C. J. Incidence algebras. New York, NY: Marcel Dekker, 1997.

Departamento de Matemática, Universidade Estadual de Maringá, Maringá — PR, CEP: 87020-900, BRAZIL

E-mail address: brusama@uem.br

Departamento de Matemática, Universidade Estadual de Maringá, Maringá — PR, CEP: 87020-900, BRAZIL

E-mail address: ezancanella@uem.br

Departamento de Matemática, Universidade Federal de Santa Catarina, Campus Reitor João David Ferreira Lima, Florianópolis - SC, CEP: 88040-900, Brazil

E-mail address: nskhripchenko@gmail.com 